\title{
Language Understanding Research at Paramax
}

\author{
Deborah A. Dahl, Carl Weir, Suzanne Liebowitz Taylor, \\ Lewis M. Norton, Marcia C. Linebarger and Mark Lipshutz * \\ Paramax Systems Corporation, a Unisys Company \\ P.O. Box 517 \\ Paoli, PA, 19301
}

\begin{abstract}
Language understanding work at Paramax focuses on applying general-purpose language understanding technology to spoken language understanding, text understanding, and document processing, integrating language understanding with speech recognition, knowledge-based information retrieval and image understanding.
\end{abstract}

\section{INTRODUCTION}

The basic language understanding technology in the Paramax language understanding architecture has been designed to be both independent of the language input modality (e.g. spoken input vs. scanned OCR input) as well as independent of the end application (e.g. command and control vs. data extraction). This architecture is shown in Figure 1.

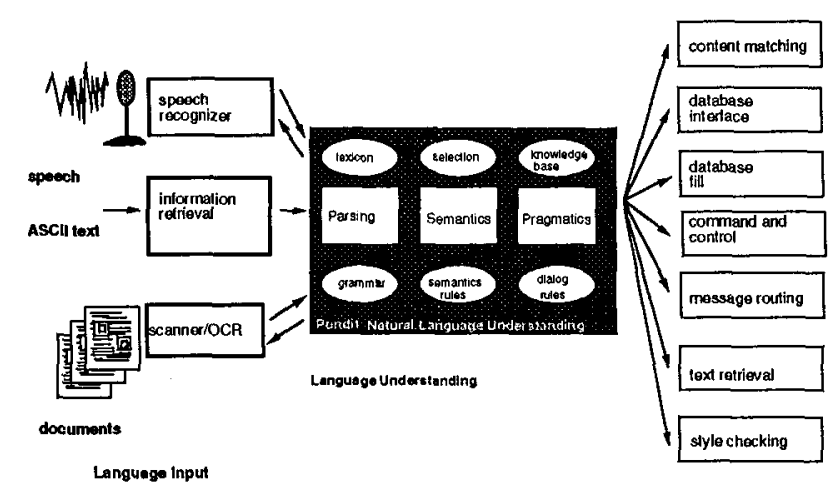

Language Applications

Figure 1: Paramax Language Understanding Inputs and Applications

\section{SPOKEN LANGUAGE UNDERSTANDING}

The focus of our spoken language understanding work is the development of a domain- and application- independent dialog processing architecture which can be coupled

- This paper was partially supported by DARPA contract N000014-89-C0171, administered by the Office of Naval Research, and by internal funding from Paramax Systems Corporation (formerly Unisys Defense Systems). with a variety of speech recognizers. Some of our recent achievements include:

- a non-monotonic reasoning capability, which will support "what if?" exploratory dialogs

- a query paraphrase component to enhance userfriendliness

- enhanced reference resolution capabilities to support additional types of context-dependent references

- automated training techniques for semantics

Figure 2 illustrates the integration of a speech recognizer, language understanding component, and a dialog manager (VFE) into a modular spoken language architecture interacting with the user and the application. A

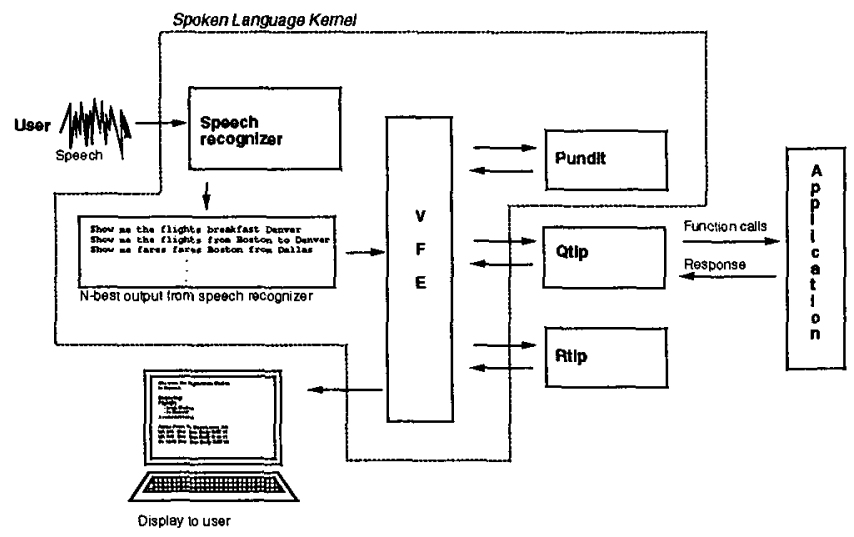

Figure 2: Paramax spoken language system architecture

more detailed description of this work can be found in [1].

\section{TEXT UNDERSTANDING}

In text understanding we emphasize an architecture in which a variety of components cooperate to produce 
an analysis of texts. Not all components may be required. for any one particular application - they can be intermixed in various ways to suit each application's needs. This architecture, shown in Figure 3 includes keyword-based information retrieval, for message routing. A knowledge-based information retrieval component (KBIRD) extracts text information which is accessable without a detailed natural language analysis. If an application requires detailed natural language processing, a natural language processing system is available. Finally, a database record generator formats the extracted information for database generation. This approach is described in [2].

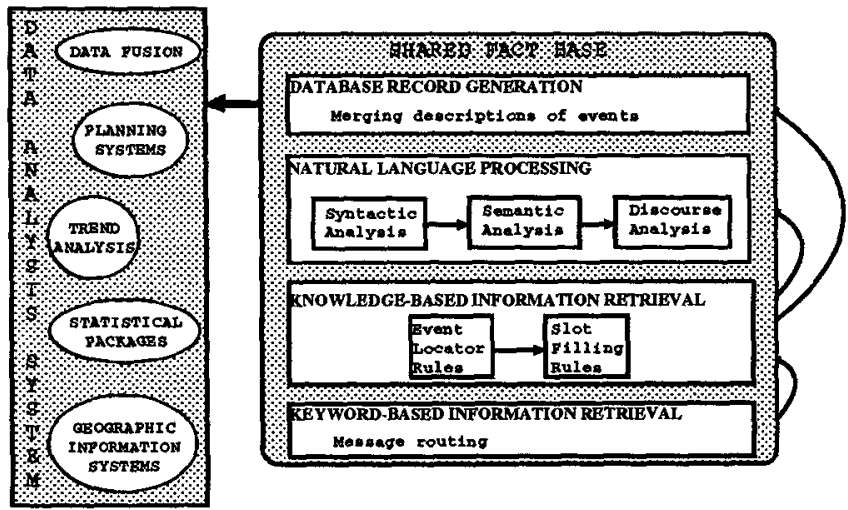

Figure 3: Paramax text understanding architecture

\section{DOCUMENT UNDERSTANDING}

The object of Paramax document understanding research is to develop novel knowledge-based approaches to the processing of document images. Starting with the scanned image(s) of a document's pages, our goal is to determine the document's functional and physical organization and to extract the key ideas from the ASCII representation of its text. Our current focus is on producing the structural interpretation of the document and the accompanying ASCII text for each component. The ASCII text would then be analyzed by a text-understanding system, as discussed in Section 2.

An intelligent document understanding system is shown in Figure 4 with the scope of our project falling in the shaded region.

This work is discussed in detail in [3].

\section{References}

1. L. M. Norton, D. A. Dahl, and M. C. Linebarger, "Recent improvements and benchmark results for the Paramax ATIS system," in Proceedings of the DARPA Speech and Language Workshop, (Harriman, New York), February 1992 .

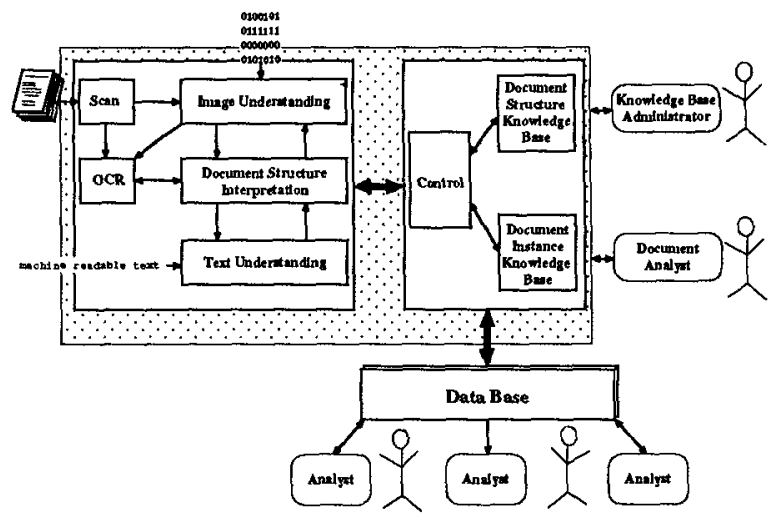

Figure 4: Multiple sources of knowledge are integrated in this document processing architecture.

2. T. Finin, R. McEntire, C. Weir, and B. Silk. "A threetiered approach to natural language text retrieval,", July 1991. AAAI-91 Workshop Program.

3. S. Liebowitz Taylor, M. Lipshutz, and C. Weir, "Document structure interpretation by integrating multiple knowledge sources," in Proceedings of the Symposium on Document Analysis and Information Retrieval, March 1992. 